\title{
Strong Convergence of the Iterative Methods for Hierarchical Fixed Point Problems of an Infinite Family of Strictly Nonself Pseudocontractions
}

\author{
Wei $\mathrm{Xu}^{1}$ and Yuanheng Wang ${ }^{2}$ \\ ${ }^{1}$ Tongji Zhejiang College, Zhejiang 314000, China \\ ${ }^{2}$ Department of Mathematics, Zhejiang Normal University, Zhejiang 321004, China \\ Correspondence should be addressed to Yuanheng Wang, wangyuanheng@yahoo.com.cn \\ Received 20 August 2012; Accepted 11 September 2012 \\ Academic Editor: Yonghong Yao
}

Copyright (C) 2012 W. Xu and Y. Wang. This is an open access article distributed under the Creative Commons Attribution License, which permits unrestricted use, distribution, and reproduction in any medium, provided the original work is properly cited.

This paper deals with a new iterative algorithm for solving hierarchical fixed point problems of an infinite family of pseudocontractions in Hilbert spaces by $y_{n}=\beta_{n} S x_{n}+\left(1-\beta_{n}\right) x_{n}, x_{n+1}=$ $P_{C}\left[\alpha_{n} f\left(x_{n}\right)+\left(1-\alpha_{n}\right) \sum_{i=1}^{\infty} \mu_{i}^{(n)} T_{i} y_{n}\right]$, and $\forall n \geq 0$, where $T_{i}: C \mapsto H$ is a nonself $k_{i}$-strictly pseudocontraction. Under certain approximate conditions, the sequence $\left\{x_{n}\right\}$ converges strongly to $x^{*} \in \bigcap_{i=1}^{\infty} F\left(T_{i}\right)$, which solves some variational inequality. The results here improve and extend some recent results.

\section{Introduction}

Let $H$ be a real Hilbert space with inner product $\langle\cdot\rangle$ and norm $\|\cdot\|$. Let $C$ be a nonempty closed convex subset of $H$. A mapping $f: C \mapsto H$ is called a contraction with coefficient $\gamma$ if there exits a constant $\gamma \in[0,1)$ such that

$$
\|f(x)-f(y)\| \leq r\|x-y\|, \quad \forall x, y \in C .
$$

A mapping $T: C \mapsto C$ is called nonexpansive if

$$
\|T x-T y\| \leq\|x-y\|, \quad \forall x, y \in C .
$$

A mapping $T: C \mapsto H$ is called $k$-strictly pseudocontraction if there exits a constant $k \in[0,1)$ such that

$$
\|T x-T y\|^{2} \leq\|x-y\|^{2}+k\|(I-T) x-(I-T) y\|^{2}, \quad \forall x, y \in C .
$$


Write $F(T)$ as the set of fixed points of $T$, that is, $F(T)=\{x \in C, T x=x\}$. In 2000, Moudafi [1] introduced an iterative scheme for nonexpansive mappings

$$
x_{n+1}=\alpha_{n} f\left(x_{n}\right)+\left(1-\alpha_{n}\right) T x_{n}, \quad \forall n \geq 0,
$$

where $f$ be a contraction on $H$ and the sequence $\left\{x_{n}\right\}$ started with arbitrary initial $x_{0} \in H$. In 2004, Xu [2] proved that the sequence $\left\{x_{n}\right\}$ generated by (1.4) converges strongly to a fixed point of $T$ under certain conditions on the parameters, which also solves the variational inequality

$$
\left\langle(I-f) x^{*}, x-x^{*}\right\rangle \geq 0, \quad \forall x \in F(T) .
$$

Recently, some authors studied the problems of fixed points of nonexpansive mappings with strongly positive operators, Lipschitizian, strongly monotone operators, and extragradient methods, and many convergence results were obtained (such as, see [3-9]).

In 2008, Yao et al. [10] introduced the following iterative scheme:

$$
\begin{gathered}
x_{0}=x \in C, \\
y_{n}=\beta_{n} x_{n}+\left(1-\beta_{n}\right) T x_{n}, \\
x_{n+1}=\alpha_{n} f\left(x_{n}\right)+\left(1-\alpha_{n}\right) y_{n}, \quad \forall n \geq 0,
\end{gathered}
$$

where $f$ is a contraction on $C$ and $T: C \mapsto C$ is nonexpansive mapping. In 2012, Song et al. [11] analyzed the following iterative algorithm:

$$
\begin{gathered}
x_{0}=x \in C, \\
y_{n}=P_{C}\left[\beta_{n} x_{n}+\left(1-\beta_{n}\right) \sum_{i=1}^{\infty} \mu_{i}^{(n)} T_{i} x_{n}\right], \\
x_{n+1}=\alpha_{n} f\left(x_{n}\right)+\gamma_{n} x_{n}+\left(\left(1-\gamma_{n}\right) I-\alpha_{n} F\right) y_{n}, \quad \forall n \geq 0,
\end{gathered}
$$

where $T_{i}$ is a $k_{i}$-strictly pseudocontraction, $F: C \mapsto C$ is a lipschitzian and strongly monotone operator, $f: C \mapsto C$ is a contraction, and $P_{C}$ is the metric projection from $H$ onto $C$. Under certain conditions on the parameters, the sequence $\left\{x_{n}\right\}$ generated by (1.7) converges strongly to a fixed point of a countable family of $k_{i}$-strictly pseudocontraction, which is the solution of some variational inequality.

On the other hand, in 2010, Yao et al. [12] introduced the iterative algorithm for solving hierarchical fixed point of nonexpansive mappings and gave the following theorem.

\section{Theorem YCL}

Let $C$ be a nonempty closed convex subset of a real Hilbert space $H$. Let $f: C \mapsto H$ be a contraction with coefficient $\gamma \in[0,1)$. Suppose the following conditions are satisfied:

(i) $\lim _{n \rightarrow \infty} \alpha_{n}=0$ and $\sum_{n=0}^{\infty} \alpha_{n}=\infty$;

(ii) $\lim _{n \rightarrow \infty}\left(\beta_{n} / \alpha_{n}\right)=0$;

(iii) $\lim _{n \rightarrow \infty}\left(\left|\alpha_{n+1}-\alpha_{n}\right| / \alpha_{n}\right)=0$ and $\lim _{n \rightarrow \infty}\left(\left|\beta_{n+1}-\beta_{n}\right| / \beta_{n}\right)=0$. 
Then the sequence $\left\{x_{n}\right\}$ generated by

$$
\begin{gathered}
x_{0}=x \in C, \\
y_{n}=\beta_{n} S x_{n}+\left(1-\beta_{n}\right) x_{n}, \\
x_{n+1}=P_{C}\left[\alpha_{n} f\left(x_{n}\right)+\left(1-\alpha_{n}\right) T y_{n}\right], \quad \forall n \geq 0,
\end{gathered}
$$

converges strongly to a point of $x^{*} \in H$, which is the unique solution of the variational inequality

$$
x^{*} \in F(T), \quad\left\langle(I-f) x^{*}, x-x^{*}\right\rangle \geq 0, \quad \forall x \in F(T) .
$$

Motivated and inspired by the iterative schemes (1.7) and (1.8), we introduce and study the hybrid iterative algorithm for solving some hierarchical fixed point problem of infinite family of strictly nonself pseudocontractions:

$$
\begin{gathered}
x_{0}=x \in C, \\
y_{n}=\beta_{n} S x_{n}+\left(1-\beta_{n}\right) x_{n} \\
x_{n+1}=P_{C}\left[\alpha_{n} f\left(x_{n}\right)+\left(1-\alpha_{n}\right) \sum_{i=1}^{\infty} \mu_{i}^{(n)} T_{i} y_{n}\right], \quad \forall n \geq 0,
\end{gathered}
$$

where $S, f$, and $P_{C}$ are the same in (1.8), $T_{i}: C \mapsto H$ is a nonself $k_{i}$-strictly pseudocontraction. Under certain conditions on the parameters, the sequence $\left\{x_{n}\right\}$ generated by (1.10) converges strongly to a common fixed point of infinite family of $k_{i}$-strictly pseudocontractions, which solves the variational inequality

$$
x^{*} \in \bigcap_{i=1}^{\infty} F\left(T_{i}\right), \quad\left\langle(I-f) x^{*}, x-x^{*}\right\rangle \geq 0, \quad \forall x \in \bigcap_{i=1}^{\infty} F\left(T_{i}\right) .
$$

So, our results extend and improve some results of other authors (such as [10-12]) from self-mappings to nonself-mappings, from nonexpansive mappings to $k_{i}$-strictly pseudocontraction, and from one mapping to a infinite family mappings.

\section{Preliminaries}

In this section, we recall some basic facts that will be needed in the proof of the main results.

Lemma 2.1 (see [13] demiclosedness principle). Let $C$ be a nonempty closed convex subset of a real Hilbert space $H$ and let $T: C \mapsto C$ be a nonexpansive mapping with $F(T) \neq \emptyset$. If $\left\{x_{n}\right\}$ is a sequence in $C$ weakly converging to $x$ and if $\left\{(I-T) x_{n}\right\}$ converges strongly to $y$, then $(I-T) x=y$; in particular if $y=0$, then $x \in F(T)$.

Lemma 2.2 (see [9]). Let $x \in H$ and $z \in C$ be any points. The following results hold:

(1) that $z=P_{C} x$ if and only if there holds the relation:

$$
\langle x-y, y-z\rangle \leq 0, \quad \forall y \in C
$$


(2) that $z=P_{C} x$ if and only if there holds the relation:

$$
\|x-z\|^{2} \leq\|x-y\|^{2}-\|y-z\|^{2}, \quad \forall y \in C ;
$$

(3) there holds the ration:

$$
\left\langle P_{C} x-P_{C} y, x-y\right\rangle \geq\left\|P_{C} x-P_{C} y\right\|^{2}, \quad \forall x, y \in H .
$$

Lemma 2.3 (see [14]). For all $x, y \in H$, the following inequality holds:

$$
\|x+y\|^{2} \leq\|x\|^{2}+2\langle y, x+y\rangle \text {. }
$$

Lemma 2.4 (see [3]). Let $f: C \mapsto H$ be a contraction with coefficient $\gamma \in[0,1)$ and let $T: C \mapsto C$ be a nonexpansive mapping. Then for all $x, y \in C$ :

(1) the mapping $(I-f)$ is strongly monotone with coefficient $(1-\gamma)$, that is,

$$
\langle x-y,(I-f) x-(I-f) y\rangle \geq(1-\gamma)\|x-y\|^{2},
$$

(2) the mapping $(I-T)$ is monotone:

$$
\left\langle x-y_{,}(I-T) x-(I-T) y\right\rangle \geq 0 .
$$

Lemma 2.5 (see [15]). Let $H$ be a Hilbert space and let $C$ be a nonempty convex subset of $H$. Let $T: C \mapsto H$ be a $k$-strictly pseudocontractive mapping with $F(T) \neq \emptyset$. Then $F\left(P_{C} T\right)=F(T)$.

Lemma 2.6 (see [16]). Let $H$ be a Hilbert space and let $C$ be a nonempty convex subset of $H$. Let $T: C \mapsto H$ be a $k$-strictly pseudocontractive mapping. Define a mapping $J x=\delta x+(1-\delta) T x$ for all $x \in C$. Then as $\delta \in[k, 1), J$ is a nonexpansive mapping such that $F(J)=F(T)$.

Lemma 2.7 (see [11]). Let $H$ be a Hilbert space and let $C$ be a nonempty convex subset of $H$. Assume that $T_{i}: C \mapsto H$ is a countable family of $k_{i}$-strictly pseudocontraction for some $0 \leq k_{i}<1$ and $\sup \left\{k_{i}: i \in N\right\}<1$ such that $\bigcap_{i=1}^{\infty} F\left(T_{i}\right) \neq \emptyset$. Assume that $\left\{\mu_{i}\right\}$ is a positive sequence such that $\sum_{i=1}^{\infty} \mu_{i}=1$. Then $\sum_{i=1}^{\infty} \mu_{i} T_{i}: C \mapsto H$ is a $k$-strictly pseudocontraction with coefficient $k=\sup \left\{k_{i}\right.$ : $i \in N\}$ and $F\left(\sum_{i=1}^{\infty} \mu_{i} T_{i}\right)=\bigcap_{i=1}^{\infty} F\left(T_{i}\right)$.

Lemma 2.8 (see [17]). Let $\left\{\alpha_{n}\right\}$ be a sequence of nonnegative real numbers satisfying the following relation: $\alpha_{n+1} \leq\left(1-\gamma_{n}\right) \alpha_{n}+\delta_{n}$, where (1) $\left\{\gamma_{n}\right\} \subset(0,1), \Sigma_{n=1}^{\infty} \gamma_{n}=\infty ;(2) \lim \sup _{n \rightarrow \infty}\left(\delta_{n} / \gamma_{n}\right) \leq 0$ or $\sum_{n=1}^{\infty}\left|\delta_{n}\right|<\infty$, then $\lim _{n \rightarrow \infty} \alpha_{n}=0$.

\section{Main Results}

In this section, we prove some strong convergence results on the iterative algorithm for solving hierarchical fixed point problem. 
Theorem 3.1. Let $H$ be a real Hilbert space and let $C$ be a nonempty closed convex subset of $H$. Let $f: C \mapsto H$ be a (possibly nonself) contraction with coefficient $\gamma \in[0,1)$, and let $S: C \mapsto C$ be a nonexpansive mapping. Let $T_{i}: C \mapsto H$ be a countable family of $k_{i}$-strictly (possibly nonself) pseudocontraction with $0 \leq k_{i} \leq k<1$ such that $\bigcap_{i=1}^{\infty} F\left(T_{i}\right) \neq \emptyset$. Let the sequence $\left\{x_{n}\right\}$ be generated by (1.10) with $\left\{\alpha_{n}\right\},\left\{\beta_{n}\right\}$ in $[0,1]$. Suppose for each $n, \sum_{i=1}^{\infty} \mu_{i}^{(n)}=1$, for all $n$ and $\mu_{i}^{(n)} \geq 0$, for all $i \in N$. Assume that the parameters satisfy the following conditions:

(i) $\lim _{n \rightarrow \infty} \alpha_{n}=0$ and $\sum_{n=0}^{\infty} \alpha_{n}=\infty$;

(ii) $\lim _{n \rightarrow \infty}\left(\beta_{n} / \alpha_{n}\right)=0$;

(iii) $\lim _{n \rightarrow \infty}\left(\left|\alpha_{n+1}-\alpha_{n}\right| / \alpha_{n}\right)=0, \lim _{n \rightarrow \infty}\left(\left|\beta_{n+1}-\beta_{n}\right| / \alpha_{n}\right)=0$, and $\lim _{n \rightarrow \infty}\left(\sum_{i=1}^{\infty} \mid \mu_{i}^{(n)}-\right.$ $\left.\mu_{i}^{(n-1)} \mid / \alpha_{n}\right)=0$

(iv) $\lim _{n \rightarrow \infty} \sum_{i=1}^{\infty}\left|\mu_{i}^{(n)}-\mu_{i}\right|=0$ and $\sum_{i=1}^{\infty} \mu_{i}=1\left(\mu_{i}>0\right)$.

Then the sequence $\left\{x_{n}\right\}$ converges strongly to $x^{*} \in \bigcap_{i=1}^{\infty} F\left(T_{i}\right)$, which solves the variational inequality

$$
\left\langle(I-f) x^{*}, x-x *\right\rangle \geq 0, \quad \forall x \in \bigcap_{i=1}^{\infty} F\left(T_{i}\right)
$$

Proof. The proof is divided into four steps.

Step 1 . We show that the sequences $\left\{x_{n}\right\}$ and $\left\{y_{n}\right\}$ are bounded.

For each $n \geq 0$, write $B_{n}=\sum_{i=1}^{\infty} \mu_{i}^{(n)} T_{i}$ and by Lemma 2.7, we have $B_{n}$ is a $k$-strictly pseudocontraction on $C$ and $F\left(B_{n}\right)=\bigcap_{i=1}^{\infty} F\left(T_{i}\right)$, for all $n \in N$. Therefore, the iterative algorithm (1.10) can be written as

$$
\begin{gathered}
x_{0}=x \in C, \\
y_{n}=\beta_{n} S x_{n}+\left(1-\beta_{n}\right) x_{n}, \\
x_{n+1}=P_{C}\left[\alpha_{n} f\left(x_{n}\right)+\left(1-\alpha_{n}\right) B_{n} y_{n}\right], \quad \forall n \geq 0 .
\end{gathered}
$$

By condition (ii), without loss of generality, we may assume $\beta_{n} \leq \alpha_{n}$, for all $n \geq 0$. Take $p \in \bigcap_{i=1}^{\infty} F\left(T_{i}\right)$ and we estimate $\left\|B_{n} y_{n}-p\right\|$. For fixed approximate $\delta \in[k, 1)$, define a mapping $J x=\delta x+(1-\delta) B_{n} x$ and by Lemma 2.6, $J$ is a nonexpansive mapping and $\operatorname{Fix}(J)=\operatorname{Fix}\left(B_{n}\right)$. So

$$
\begin{aligned}
\left\|B_{n} y_{n}-p\right\| & =\left\|\frac{1}{1-\delta}\left(J y_{n}-\delta y_{n}\right)-p\right\| \\
& \leq \frac{1}{1-\delta}\left(\left\|J y_{n}-J p\right\|+\delta\left\|y_{n}-p\right\|\right) \\
& \leq \frac{1+\delta}{1-\delta}\left\|y_{n}-p\right\| .
\end{aligned}
$$


Together with (3.2) and (3.3), we get

$$
\begin{aligned}
\left\|x_{n+1}-p\right\|= & \left\|P_{C}\left[\alpha_{n} f\left(x_{n}\right)+\left(1-\alpha_{n}\right) B_{n} y_{n}\right]-P_{C} p\right\| \\
\leq & \left\|\alpha_{n} f\left(x_{n}\right)+\left(1-\alpha_{n}\right) B_{n} y_{n}-p\right\| \\
\leq & \alpha_{n}\left\|f\left(x_{n}\right)-f(p)\right\|+\alpha_{n}\|f(p)-p\|+\left(1-\alpha_{n}\right)\left\|B_{n} y_{n}-p\right\| \\
\leq & \alpha_{n} \gamma\left\|x_{n}-p\right\|+\alpha_{n}\|f(p)-p\|+\left(1-\alpha_{n}\right) \frac{1+\delta}{1-\delta}\left\|y_{n}-p\right\| \\
\leq & \alpha_{n} \gamma\left\|x_{n}-p\right\|+\alpha_{n}\|f(p)-p\|+\left(1-\alpha_{n}\right) \frac{1+\delta}{1-\delta} \beta_{n}\left\|S x_{n}-p\right\| \\
& +\left(1-\alpha_{n}\right) \frac{1+\delta}{1-\delta}\left(1-\beta_{n}\right)\left\|x_{n}-p\right\| \\
\leq & \alpha_{n} \gamma\left\|x_{n}-p\right\|+\alpha_{n}\|f(p)-p\|+\left(1-\alpha_{n}\right) \frac{1+\delta}{1-\delta} \beta_{n}\left\|S x_{n}-S p\right\| \\
& +\left(1-\alpha_{n}\right) \frac{1+\delta}{1-\delta} \beta_{n}\|S p-p\|+\left(1-\alpha_{n}\right) \frac{1+\delta}{1-\delta}\left(1-\beta_{n}\right)\left\|x_{n}-p\right\| \\
\leq & \alpha_{n} \gamma\left\|x_{n}-p\right\|+\left(1-\alpha_{n}\right) \frac{1+\delta}{1-\delta}\left\|x_{n}-p\right\|+\frac{1+\delta}{1-\delta} \alpha_{n}\|S p-p\|+\alpha_{n}\|f(p)-p\| \\
= & {\left[1-\alpha_{n}\left(\frac{1+\delta}{1-\delta}-\gamma\right)\right]\left\|x_{n}-p\right\| } \\
& \left.+\frac{1+\delta}{1-\delta}-\gamma\right)\left[\frac{1}{((1+\delta) /(1-\delta))-\gamma}\left(\frac{1+\delta}{1-\delta}\|S p-p\|+\|f(p)-p\|\right)\right] .
\end{aligned}
$$

Therefore, we obtain

$$
\left\|x_{n}-p\right\| \leq \max \left\{\left\|x_{0}-p\right\|, \frac{1}{((1+\delta) /(1-\delta))-\gamma}\left(\frac{1+\delta}{1-\delta}\|S p-p\|+\|f(p)-p\|\right)\right\},
$$

which gives the results that the sequence $\left\{x_{n}\right\}$ is bounded and so are $\left\{f\left(x_{n}\right)\right\},\left\{y_{n}\right\},\left\{B_{n} x_{n}\right\}$, $\left\{B_{n} y_{n}\right\}$.

Step 2. Now we show that $\left\|x_{n+1}-x_{n}\right\| \rightarrow 0$ as $n \rightarrow \infty$. Let

$$
v_{n}=\alpha_{n} f\left(x_{n}\right)+\left(1-\alpha_{n}\right) B_{n} y_{n}
$$

Next we estimate $\left\|x_{n+1}-x_{n}\right\|$. From (3.2), we have

$$
\begin{aligned}
\left\|x_{n+1}-x_{n}\right\| & =\left\|P_{C}\left[v_{n}\right]-P_{C}\left[v_{n-1}\right]\right\| \\
\leq & \left\|v_{n}-v_{n-1}\right\| \\
& =\left\|\alpha_{n} f\left(x_{n}\right)+\left(1-\alpha_{n}\right) B_{n} y_{n}-\alpha_{n-1} f\left(x_{n-1}\right)-\left(1-\alpha_{n-1}\right) B_{n-1} y_{n-1}\right\| \\
& =\| \alpha_{n}\left(f\left(x_{n}\right)-f\left(x_{n-1}\right)\right)+\left(\alpha_{n}-\alpha_{n-1}\right) f\left(x_{n-1}\right)+\left(1-\alpha_{n}\right)\left(B_{n} y_{n}-B_{n} y_{n-1}\right) \\
& \quad+\left(1-\alpha_{n}\right) B_{n} y_{n-1}+\left(1-\alpha_{n-1}\right)\left(B_{n} y_{n-1}-B_{n-1} y_{n-1}\right) \|
\end{aligned}
$$


Abstract and Applied Analysis

$$
\begin{aligned}
\leq & \alpha_{n}\left\|f\left(x_{n}\right)-f\left(x_{n-1}\right)\right\|+\left|\alpha_{n}-\alpha_{n-1}\right|\left\|f\left(x_{n-1}\right)-B_{n} y_{n-1}\right\| \\
& +\left(1-\alpha_{n}\right) \frac{1+\delta}{1-\delta}\left\|y_{n}-y_{n-1}\right\|+\left(1-\alpha_{n-1}\right) \sum_{i=1}^{\infty}\left|\mu_{i}^{(n)}-\mu_{i}^{(n-1)}\right|\left\|T_{i} y_{n-1}\right\| \\
\leq & \alpha_{n} \gamma\left\|x_{n}-x_{n-1}\right\|+\left(1-\alpha_{n}\right) \frac{1+\delta}{1-\delta}\left\|y_{n}-y_{n-1}\right\| \\
& +M\left[\left|\alpha_{n}-\alpha_{n-1}\right|+\sum_{i=1}^{\infty}\left|\mu_{i}^{(n)}-\mu_{i}^{(n-1)}\right|\right]
\end{aligned}
$$

where $M$ is a constant such that

$$
\sup _{n \in N}\left\{\left\|f\left(x_{n-1}\right)-B_{n} y_{n-1}\right\|+\left\|S\left(x_{n-1}\right)-x_{n-1}\right\|+\left\|T_{i} y_{n-1}\right\|\right\} \leq M
$$

From (3.2), we also obtain

$$
\begin{aligned}
\left\|y_{n}-y_{n-1}\right\|= & \left\|\beta_{n} S x_{n}+\left(1-\beta_{n}\right) x_{n}-\beta_{n-1} S x_{n-1}-\left(1-\beta_{n-1}\right) x_{n-1}\right\| \\
= & \| \beta_{n}\left(S x_{n}-S x_{n-1}\right)+\left(\beta_{n}-\beta_{n-1}\right) S x_{n-1} \\
& \quad+\left(1-\beta_{n}\right)\left(x_{n}-x_{n-1}\right)+\left(\beta_{n-1}-\beta_{n}\right) x_{n-1} \| \\
= & \left\|\beta_{n}\left(S x_{n}-S x_{n-1}\right)+\left(1-\beta_{n}\right)\left(x_{n}-x_{n-1}\right)+\left(\beta_{n}-\beta_{n-1}\right)\left(S x_{n-1}-x_{n-1}\right)\right\| \\
\leq & \left\|x_{n}-x_{n-1}\right\|+\left|\beta_{n}-\beta_{n-1}\right| M .
\end{aligned}
$$

Together with (3.7) and (3.9), we have

$$
\begin{aligned}
\left\|x_{n+1}-x_{n}\right\| \leq & \left\|v_{n}-v_{n-1}\right\| \\
\leq & \alpha_{n} \gamma\left\|x_{n}-x_{n-1}\right\|+\left(1-\alpha_{n}\right) \frac{1+\delta}{1-\delta}\left\|x_{n}-x_{n-1}\right\|+\left(1-\alpha_{n}\right)\left|\beta_{n}-\beta_{n-1}\right| \frac{1+\delta}{1-\delta} M \\
& +\left|\alpha_{n}-\alpha_{n-1}\right| M+\left(1-\alpha_{n-1}\right) \sum_{i=1}^{\infty}\left|\mu_{i}^{(n)}-\mu_{i}^{(n-1)}\right| M \\
\leq & {\left[1-\alpha_{n}\left(\frac{1+\delta}{1-\delta}-\gamma\right)\right]\left\|x_{n}-x_{n-1}\right\| } \\
& +M\left[\left|\alpha_{n}-\alpha_{n-1}\right|+\left|\beta_{n}-\beta_{n-1}\right| \frac{1+\delta}{1-\delta}+\sum_{i=1}^{\infty}\left|\mu_{i}^{(n)}-\mu_{i}^{(n-1)}\right|\right]
\end{aligned}
$$




$$
\begin{aligned}
= & {\left[1-\alpha_{n}\left(\frac{1+\delta}{1-\delta}-\gamma\right)\right]\left\|x_{n}-x_{n-1}\right\|+M\left(\alpha_{n}\left(\frac{1+\delta}{1-\delta}-\gamma\right)\right) } \\
\times & \times \frac{1}{\alpha_{n}(((1+\delta) /(1-\delta))-\gamma)} \\
& \left.\quad \times\left(\left|\alpha_{n}-\alpha_{n-1}\right|+\left|\beta_{n}-\beta_{n-1}\right| \frac{1+\delta}{1-\delta}+\sum_{i=1}^{\infty}\left|\mu_{i}^{(n)}-\mu_{i}^{(n-1)}\right|\right)\right] .
\end{aligned}
$$

By Lemma 2.8 and conditions (i)-(iii), we immediately get $\left\|x_{n+1}-x_{n}\right\| \rightarrow 0$ as $n \rightarrow \infty$.

Step 3 . Next we prove that $\left\|x_{n}-P_{C}\left(\sum_{i=1}^{\infty} \mu_{i} T_{i}\right) x_{n}\right\| \rightarrow 0$ as $n \rightarrow \infty$.

Let $B=\sum_{i=1}^{\infty} \mu_{i} T_{i}$. By Lemma 2.7 and condition (iv), we get the results that $B: C \mapsto H$ is a $k$-strictly pseudocontraction with $F(B)=\bigcap_{i=1}^{\infty} F\left(T_{i}\right)$ and $B_{n} x \rightarrow B x$ as $n \rightarrow \infty$, for any $x \in C$,

$$
\begin{aligned}
\left\|x_{n}-P_{C} B x_{n}\right\| \leq & \left\|x_{n}-x_{n+1}\right\|+\left\|x_{n+1}-P_{C} B x_{n}\right\| \\
= & \left\|x_{n}-x_{n+1}\right\|+\left\|P_{C}\left[v_{n}\right]-P_{C} B x_{n}\right\| \\
\leq & \left\|x_{n}-x_{n+1}\right\|+\left\|\alpha_{n} f\left(x_{n}\right)+\left(1-\alpha_{n}\right) B_{n} y_{n}-B_{n} x_{n}\right\|+\left\|B_{n} x_{n}-B x_{n}\right\| \\
= & \left\|x_{n}-x_{n+1}\right\|+\left\|\alpha_{n}\left(f\left(x_{n}\right)-B_{n} x_{n}\right)+\left(1-\alpha_{n}\right)\left(B_{n} y_{n}-B_{n} x_{n}\right)\right\| \\
& +\left\|B_{n} x_{n}-B x_{n}\right\| \\
\leq & \left\|x_{n}-x_{n+1}\right\|+\alpha_{n}\left\|f\left(x_{n}\right)-B_{n} x_{n}\right\| \\
& +\left(1-\alpha_{n}\right) \frac{1+\delta}{1-\delta}\left\|y_{n}-x_{n}\right\|+\left\|B_{n} x_{n}-B x_{n}\right\| \\
= & \left\|x_{n}-x_{n+1}\right\|+\alpha_{n}\left\|f\left(x_{n}\right)-B_{n} x_{n}\right\| \\
& +\left(1-\alpha_{n}\right) \frac{1+\delta}{1-\delta} \beta_{n}\left\|S x_{n}-x_{n}\right\|+\left\|B_{n} x_{n}-B x_{n}\right\| .
\end{aligned}
$$

Because $\alpha_{n} \rightarrow 0, \beta_{n} \rightarrow 0,\left\|x_{n+1}-x_{n}\right\| \rightarrow 0$, and $B_{n} x \rightarrow B x$, so we obtain $\left\|x_{n}-\sum_{i=1}^{\infty} \mu_{i} T_{i} x_{n}\right\| \rightarrow$ 0 as $n \rightarrow \infty$.

Step 4 . Now we show that $\lim _{\sup _{n \rightarrow \infty}}\left\langle f\left(x^{*}\right)-x^{*}, x_{n}-x^{*}\right\rangle \leq 0$, where $x^{*}=P_{F(B)} f\left(x^{*}\right)$.

Since the sequence $\left\{x_{n}\right\}$ is bounded, we take $s$ subsequence $\left\{x_{n_{k}}\right\}$ of $\left\{x_{n}\right\}$ such that $\limsup _{n \rightarrow \infty}\left\langle f\left(x^{*}\right)-x^{*}, x_{n}-x^{*}\right\rangle=\lim _{k \rightarrow \infty}\left\langle f\left(x^{*}\right)-x^{*}, x_{n_{k}}-x^{*}\right\rangle$ and $x_{n_{k}} \rightarrow x^{\prime}$. Notice that $\left\|x_{n}-P_{C} B x_{n}\right\| \rightarrow 0$ and by Lemmas 2.1 and 2.5, we have $x^{\prime} \in \operatorname{Fix}\left(P_{C} B\right)=F(B)=\bigcap_{i=1}^{\infty} F\left(T_{i}\right)$. Then

$$
\lim _{k \rightarrow \infty}\left\langle f\left(x^{*}\right)-x^{*}, x_{n_{k}}-x^{*}\right\rangle=\left\langle f\left(x^{*}\right)-x^{*}, x^{\prime}-x^{*}\right\rangle \leq 0, \quad x^{\prime} \in F(B)
$$

Now, by Lemma 2.2, we get $\left\langle P_{C}\left[v_{n}\right]-v_{n}, P_{C}\left[v_{n}\right]-x^{*}\right\rangle \leq 0$. Therefore, we have

$$
\begin{aligned}
\left\|x_{n+1}-x^{*}\right\|^{2} & =\left\langle P_{C}\left[v_{n}\right]-x^{*}, x_{n+1}-x^{*}\right\rangle \\
& =\left\langle P_{C}\left[v_{n}\right]-v_{n}, x_{n+1}-x^{*}\right\rangle+\left\langle v_{n}-x^{*}, x_{n+1}-x^{*}\right\rangle
\end{aligned}
$$


Abstract and Applied Analysis

$$
\begin{aligned}
& =\left\langle P_{C}\left[v_{n}\right]-v_{n}, P_{C}\left[v_{n}\right]-x^{*}\right\rangle+\left\langle v_{n}-x^{*}, x_{n+1}-x^{*}\right\rangle \\
& \leq\left\langle v_{n}-x^{*}, x_{n+1}-x^{*}\right\rangle \\
& =\left\langle\alpha_{n} f\left(x_{n}\right)+\left(1-\alpha_{n}\right) B_{n} y_{n}-x^{*}, x_{n+1}-x^{*}\right\rangle \\
& =\alpha_{n}\left\langle\left(f\left(x_{n}\right)-f\left(x^{*}\right)\right), x_{n+1}-x^{*}\right\rangle+\alpha_{n}\left\langle f\left(x^{*}\right)-x^{*}, x_{n+1}-x^{*}\right\rangle \\
& +\left(1-\alpha_{n}\right)\left\langle B_{n} y_{n}-x^{*}, x_{n+1}-x^{*}\right\rangle \\
& \leq \alpha_{n} \gamma\left\|x_{n}-x^{*}\right\| \cdot\left\|x_{n+1}-x^{*}\right\|+\alpha_{n}\left\langle f\left(x^{*}\right)-x^{*}, x_{n+1}-x^{*}\right\rangle \\
& +\left(1-\alpha_{n}\right)\left\langle B_{n}\left[\beta_{n} S x_{n}+\left(1-\beta_{n}\right) x_{n}\right]-B_{n} x^{*}, x_{n+1}-x^{*}\right\rangle \\
& \leq \alpha_{n} \gamma\left\|x_{n}-x^{*}\right\| \cdot\left\|x_{n+1}-x^{*}\right\|+\alpha_{n}\left\langle f\left(x^{*}\right)-x^{*}, x_{n+1}-x^{*}\right\rangle \\
& +\left(1-\alpha_{n}\right) \| \beta_{n}\left(S x_{n}-S x^{*}\right)+\beta_{n}\left(S x^{*}-x^{*}\right) \\
& +\left(1-\beta_{n}\right)\left(x_{n}-x^{*}\right)\|\cdot\| x_{n+1}-x^{*} \| \\
& \leq \alpha_{n} \gamma\left\|x_{n}-x^{*}\right\| \cdot\left\|x_{n+1}-x^{*}\right\|+\alpha_{n}\left\langle f\left(x^{*}\right)-x^{*}, x_{n+1}-x^{*}\right\rangle \\
& +\left(1-\alpha_{n}\right)\left\|x_{n}-x^{*}\right\| \cdot\left\|x_{n+1}-x^{*}\right\|+\left(1-\alpha_{n}\right) \beta_{n}\left\|S x^{*}-x^{*}\right\| \cdot\left\|x_{n+1}-x^{*}\right\| \\
& =\left[1-(1-\gamma) \alpha_{n}\right]\left\|x_{n}-x^{*}\right\| \cdot\left\|x_{n+1}-x^{*}\right\|+\alpha_{n}\left\langle f\left(x^{*}\right)-x^{*}, x_{n+1}-x^{*}\right\rangle \\
& +\left(1-\alpha_{n}\right) \beta_{n}\left\|S x^{*}-x^{*}\right\| \cdot\left\|x_{n+1}-x^{*}\right\| \\
& \leq \frac{1-(1-\gamma) \alpha_{n}}{2}\left(\left\|x_{n}-x^{*}\right\|^{2}+\left\|x_{n+1}-x^{*}\right\|^{2}\right)+\alpha_{n}\left\langle f\left(x^{*}\right)-x^{*}, x_{n+1}-x^{*}\right\rangle \\
& +\left(1-\alpha_{n}\right) \beta_{n}\left\|S x^{*}-x^{*}\right\| \cdot\left\|x_{n+1}-x^{*}\right\| .
\end{aligned}
$$

Hence it follows that

$$
\begin{aligned}
\left\|x_{n+1}-x^{*}\right\|^{2} \leq & {\left[1-\frac{2(1-\gamma) \alpha_{n}}{1+(1-\gamma) \alpha_{n}}\right]\left\|x_{n}-x^{*}\right\|^{2}+\frac{2 \alpha_{n}}{1+(1-\gamma) \alpha_{n}}\left\langle f\left(x^{*}\right)-x^{*}, x_{n+1}-x^{*}\right\rangle } \\
& +\frac{2\left(1-\alpha_{n}\right) \beta_{n}}{1+(1-\gamma) \alpha_{n}}\left\|S x^{*}-x^{*}\right\| \cdot\left\|x_{n+1}-x^{*}\right\| \\
= & {\left[1-\frac{2(1-\gamma) \alpha_{n}}{1+(1-\gamma) \alpha_{n}}\right]\left\|x_{n}-x^{*}\right\|^{2} } \\
& +\frac{2(1-\gamma) \alpha_{n}}{1+(1-\gamma) \alpha_{n}}\left[\frac{1}{(1-\gamma)}\left\langle f\left(x^{*}\right)-x^{*}, x_{n+1}-x^{*}\right\rangle\right. \\
& \left.+\frac{\left(1-\alpha_{n}\right) \beta_{n}}{(1-\gamma) \alpha_{n}}\left\|S x^{*}-x^{*}\right\| \cdot\left\|x_{n+1}-x^{*}\right\|\right] .
\end{aligned}
$$


Now, by Lemma 2.8, conditions (i)-(iii), and

$$
\limsup _{n \rightarrow \infty}\left[\frac{1}{(1-\gamma)}\left\langle f\left(x^{*}\right)-x^{*}, x_{n+1}-x^{*}\right\rangle+\frac{\left(1-\alpha_{n}\right) \beta_{n}}{(1-\gamma) \alpha_{n}}\left\|S x^{*}-x^{*}\right\| \cdot\left\|x_{n+1}-x^{*}\right\|\right] \leq 0,
$$

we have $x_{n} \rightarrow x^{*} \in \bigcap_{i=1}^{\infty} F\left(T_{i}\right)$ as $n \rightarrow \infty$ and $x^{*}$ also solves the variational inequality

$$
\left\langle(I-f) x^{*}, x-x^{*}\right\rangle \geq 0, \quad \forall x \in \bigcap_{i=1}^{\infty} F\left(T_{i}\right) .
$$

This completes the proof.

From Theorem 3.1, if we take $S=I$ or $\beta_{n}=0$, for all $n \in N$, we get the following corollary.

Corollary 3.2. Let $H$ be a real Hilbert space and $C$ be a nonempty closed convex subset of $H$. Let $f: C \mapsto H$ be a (possibly nonself) contraction with coefficient $\gamma \in[0,1)$ and let $T_{i}: C \mapsto H$ be a countable family of $k_{i}$-strictly (possibly nonself) pseudocontraction with $0 \leq k_{i}<1$ and $\sup \left\{k_{i}: i \in\right.$ $N\}<1$ such that $\bigcap_{i=1}^{\infty} F\left(T_{i}\right) \neq \emptyset$. Let the sequence $\left\{x_{n}\right\}$ be generated by

$$
\begin{gathered}
x_{0}=x \in C, \\
x_{n+1}=P_{C}\left[\alpha_{n} f\left(x_{n}\right)+\left(1-\alpha_{n}\right) \sum_{i=1}^{\infty} \mu_{i}^{(n)} T_{i} x_{n}\right], \quad \forall n \geq 0,
\end{gathered}
$$

with $\left\{\alpha_{n}\right\}$ in $[0,1]$. Suppose for each $n, \sum_{i=1}^{\infty} \mu_{i}^{(n)}=1$, for all $n$ and $\mu_{i}^{(n)} \geq 0$, for all $i \in N$. Assume that the parameters satisfied the following conditions:

(i) $\lim _{n \rightarrow \infty} \alpha_{n}=0$ and $\sum_{n=0}^{\infty} \alpha_{n}=\infty$;

(ii) $\lim _{n \rightarrow \infty}\left(\left|\alpha_{n+1}-\alpha_{n}\right| / \alpha_{n}\right)=0$, and $\lim _{n \rightarrow \infty}\left(\left(\sum_{i=1}^{\infty}\left|\mu_{i}^{(n)}-\mu_{i}^{(n-1)}\right|\right) / \alpha_{n}\right)=0$;

(iii) $\lim _{n \rightarrow \infty} \sum_{i=1}^{\infty}\left|\mu_{i}^{(n)}-\mu_{i}\right|=0$ and $\sum_{i=1}^{\infty} \mu_{i}=1\left(\mu_{i}>0\right)$.

Then the the sequence $\left\{x_{n}\right\}$ converges strongly to $x^{*} \in \bigcap_{i=1}^{\infty} F\left(T_{i}\right)$, which solves the variational inequality

$$
\left\langle(I-f) x^{*}, x-x^{*}\right\rangle \geq 0, \quad \forall x \in \bigcap_{i=1}^{\infty} F\left(T_{i}\right) .
$$

Remark 3.3. Theorem 3.1 extends and improves Theorem YCL in the following way. The nonexpasnsive self-mapping $T: C \mapsto C$ is extended to a infinite family of nonself $k_{i}$ strictly pseudocontraction $T_{i}: C \mapsto H$. If we take $k_{i}=0, i \in N$ in Theorem 3.1, then $B=\sum_{i=1}^{\infty} \mu_{i} T_{i}$ reduces to a nonexpasnsive (possibly nonself) mapping, thus Theorem 3.1 reduces to Theorem YCL. 


\section{Acknowledgments}

The authors would like to thank editors and referees for many useful comments and suggestions for the improvement of the paper. This paper was partially supported by the Natural Science Foundation of Zhejiang Province (Y6100696) and NSFC (11071169).

\section{References}

[1] A. Moudafi, "Viscosity approximation methods for fixed-points problems," Journal of Mathematical Analysis and Applications, vol. 241, no. 1, pp. 46-55, 2000.

[2] H.-K. Xu, "Viscosity approximation methods for nonexpansive mappings," Journal of Mathematical Analysis and Applications, vol. 298, no. 1, pp. 279-291, 2004.

[3] G. Marino and H.-K. Xu, "A general iterative method for nonexpansive mappings in Hilbert spaces," Journal of Mathematical Analysis and Applications, vol. 318, no. 1, pp. 43-52, 2006.

[4] L.-C. Ceng, S.-M. Guu, and J.-C. Yao, "A general iterative method with strongly positive operators for general variational inequalities," Computers \& Mathematics with Applications. An International Journal, vol. 59, no. 4, pp. 1441-1452, 2010.

[5] M. Tian, "A general iterative algorithm for nonexpansive mappings in Hilbert spaces," Nonlinear Analysis. Theory, Methods \& Applications, vol. 73, no. 3, pp. 689-694, 2010.

[6] L.-C. Ceng, S.-M. Guu, and J.-C. Yao, "A general composite iterative algorithm for nonexpansive mappings in Hilbert spaces," Computers \& Mathematics with Applications, vol. 61, no. 9, pp. 2447-2455, 2011.

[7] Y. H. Yao and Y. C. Liou, "Composite algorithms for minimization over the solutions of equilibrium problems and fixed point problems," Abstract and Applied Analysis, vol. 2010, Article ID 763506, 19 pages, 2010.

[8] L.-C. Ceng, Q. H. Ansari, and J.-C. Yao, "Relaxed extragradient iterative methods for variational inequalities," Applied Mathematics and Computation, vol. 218, no. 3, pp. 1112-1123, 2011.

[9] Y. H. Yao, M. A. Noor, and Y. C. Liou, "Strong convergence of a modified extragradient method to the minium-norm solution of variational inequalities," Abstract and Applied Analysis, vol. 2012, Article ID Article ID 817436, 9 pages, 2012.

[10] Y. Yao, R. Chen, and J.-C. Yao, "Strong convergence and certain control conditions for modified Mann iteration," Nonlinear Analysis. Theory, Methods E Applications, vol. 68, no. 6, pp. 1687-1693, 2008.

[11] Y. L. Song, H. Y. Hu, Y. Q. Wang et al., "Strong convergence of a new general iterative method for variational inequality problems in Hilbert spaces," Fixed Point Theory and Applications, vol. 2012, 46 pages, 2012.

[12] Y. H. Yao, Y. J. Cho, and Y.-C. Liou, "Iterative algorithms for hierarchical fixed points problems and variational inequalities," Mathematical and Computer Modelling, vol. 52, no. 9-10, pp. 1697-1705, 2010.

[13] Y. H. Wang and Y. H. Xia, "Strong convergence for asymptotically pseudocontractions with the demiclosedness principle in Banach spaces," Fixed Point Theory and Applications, vol. 2012, 45 pages, 2012.

[14] S. S. Chang, Y. J. Cho, and H. Y. Zhou, Iterative Methods for Nonlinear Operator Equations in Banach Spaces, Nova Science Publisher Inc., Huntington, NY, USA, 2002.

[15] H. Y. Zhou, "Convergence theorems of fixed points for $k$-strict pseudo-contractions in Hilbert spaces," Nonlinear Analysis. Theory, Methods \& Applications, vol. 69, no. 2, pp. 456-462, 2008.

[16] F. E. Browder and W. V. Petryshyn, "Construction of fixed points of nonlinear mappings in Hilbert space," Journal of Mathematical Analysis and Applications, vol. 20, pp. 197-228, 1967.

[17] Y. H. Wang and L. Yang, "Modified relaxed extragradient method for a general system of variational inequalities and nonexpansive mappings in Banach spaces," Abstract and Applied Analysis, vol. 2012, Article ID 818970, 14 pages, 2012. 


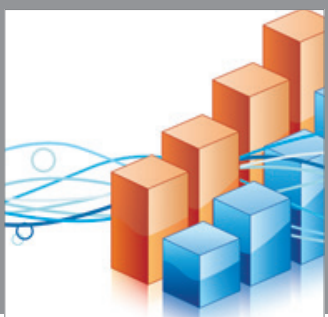

Advances in

Operations Research

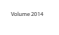

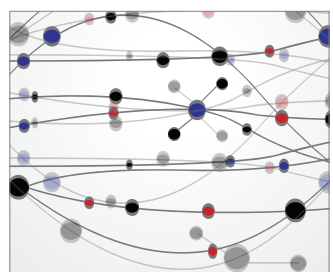

\section{The Scientific} World Journal
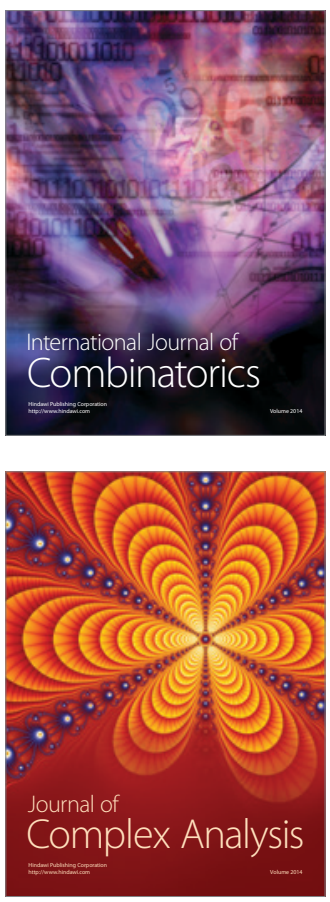

International Journal of

Mathematics and

Mathematical

Sciences
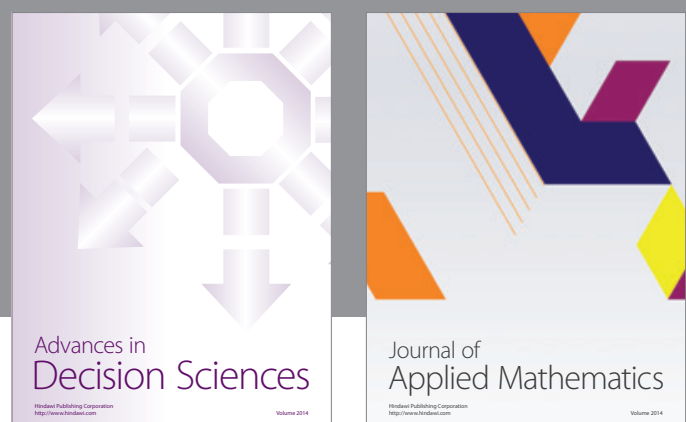

Journal of

Applied Mathematics
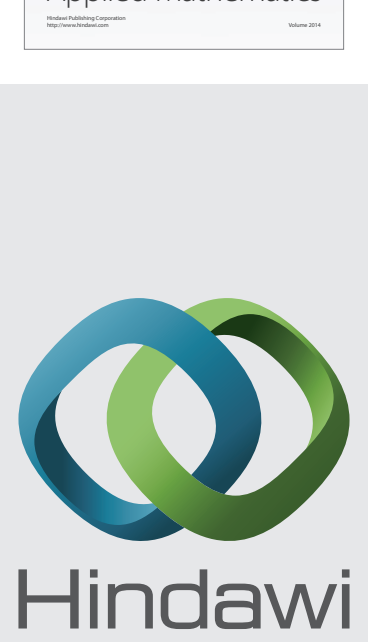

Submit your manuscripts at http://www.hindawi.com
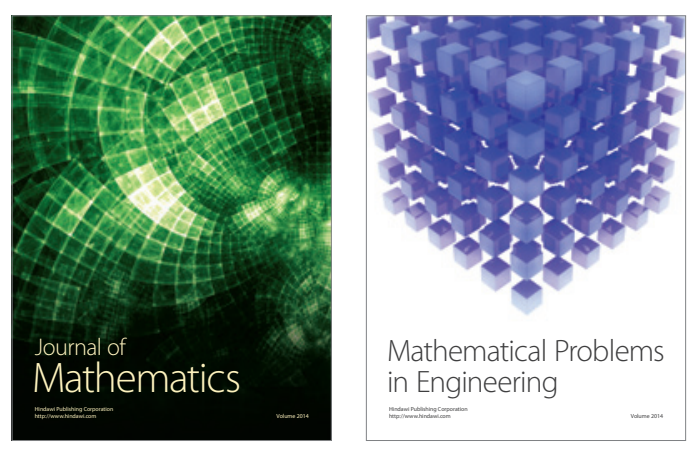

Mathematical Problems in Engineering
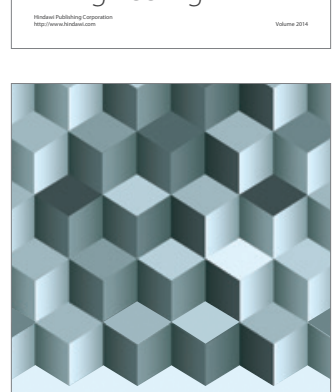

Journal of

Function Spaces
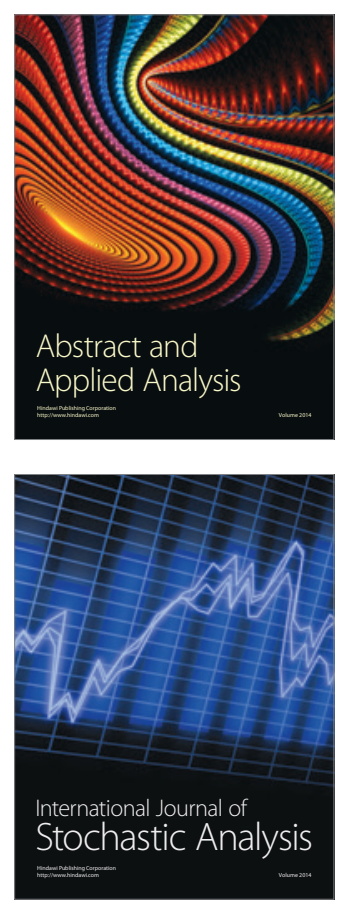

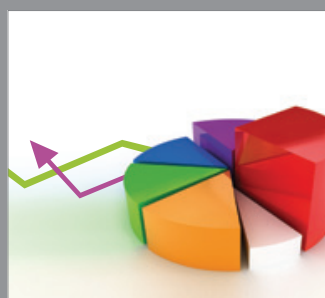

ournal of

Probability and Statistics

Promensencen
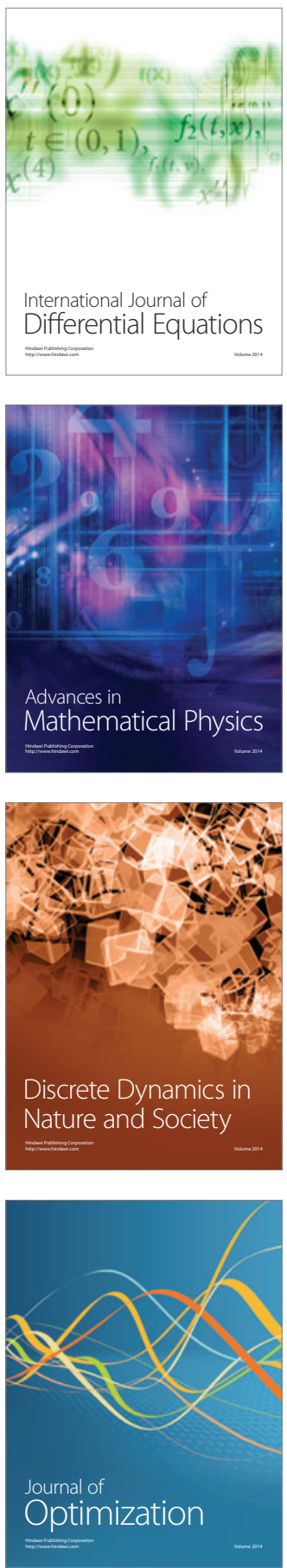